\title{
Innovation, créativité et qualité de vie au travail : proposition et expérimentation d'un dispositif d'innovation transitionnelle
}

Innovation, creativity and quality of life at work: motion and experimentation of a transitional innovation device

\section{Nadège Guidou et Philippe Sarnin}

\section{(2) OpenEdition}

\section{Journals}

Édition électronique

URL : https://journals.openedition.org/activites/6800

DOI : 10.4000/activites.6800

ISSN : 1765-2723

Éditeur

ARPACT - Association Recherches et Pratiques sur les ACTivités

\section{Référence électronique}

Nadège Guidou et Philippe Sarnin, «Innovation, créativité et qualité de vie au travail : proposition et expérimentation d'un dispositif d'innovation transitionnelle ». Activités [En ligne], 18-2 | 2021, mis en ligne le 15 octobre 2021, consulté le 07 avril 2022. URL : http://journals.openedition.org/activites/ 6800 ; DOI : https://doi.org/10.4000/activites.6800

Ce document a été généré automatiquement le 7 avril 2022.

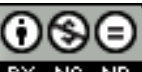

Activités est mis à disposition selon les termes de la licence Creative Commons Attribution - Pas d'Utilisation Commerciale - Pas de Modification 4.0 International. 


\title{
Innovation, créativité et qualité de vie au travail : proposition et expérimentation d'un dispositif d'innovation transitionnelle
}

\author{
Innovation, creativity and quality of life at work: motion and experimentation of \\ a transitional innovation device
}

Nadège Guidou et Philippe Sarnin

\section{NOTE DE L'ÉDITEUR}

Article soumis le 03/02/2021, accepté le 24/08/2021

\section{Introduction}

L'intérêt pour la santé psychique au travail se renouvelle sans cesse au travers des travaux de recherche, des démarches des praticiens et des entreprises ou encore des traitements médiatiques. Malgré cette combinaison d'éclairages et de volontés, des limites sont toujours observées notamment pour passer à l'action transformatrice (Becque, Mauroux, Baradji, \& Dennevault, 2017). Les sciences sociales se questionnent donc sur les moyens à mobiliser pour soutenir le processus allant du diagnostic à la transformation durable (Debard, 2015; Hansez \& De Keyser, 2007). Les entreprises cherchent elles aussi à expérimenter de nouvelles formes d'organisation (Detchessahar, 2019 ; Ughetto, 2018) pour plus d'efficacité (digitalisation, innovation, mode agile, etc.), mais aussi pour davantage de santé (développement de la qualité de vie au travail, de l'autonomie, du sens du travail, etc.). La psychologie du travail et des organisations, par ses ancrages épistémologiques et son lien toujours conservé avec le terrain (Clot, 1995), compte parmi les disciplines pouvant contribuer à ces réflexions autour de 
l'articulation entre performances économiques et sociales (Caillé \& Jeoffrion, 2017). C'est dans ce cadre que nous présentons une nouvelle méthodologie d'intervention pensée pour répondre à cette double finalité en alliant processus d'innovation organisationnelle (Alter, 2000; Battistelli, 2014) et processus transitionnel (Amado, 2015 ; Winnicott, 1971). Après avoir discuté ses fondements épistémologiques et méthodologiques, nous détaillerons sa première expérimentation réalisée en service de santé au travail dans un contexte d'intervention dégradé. En nous plaçant du côté de la clinique de l'intervention, nous mettrons particulièrement en lumière le processus d'intervention puis tenterons d'en induire les apports et les limites.

\section{Du soutien aux transformations à l'accompagnement des acteurs aux prises avec ces transformations}

2 La nouvelle dénomination politique des démarches de prévention de la santé psychique sous le nom de qualité de vie au travail (QVT) est utilisée pour signifier que les objectifs sont de combiner santé des salariés et performance de l'entreprise (Grosjean \& Guyot, 2016; Supiot, 2017). Or, les sciences du travail, et particulièrement l'ergonomie (Sznelwar, \& Le Doaré, 2006), la psychologie du travail (Caillé, Courtois, Galharret, \& Jeoffrion, 2020 ; Fucsh, Jeoffrion, Barré, Savary, 2018) et plus récemment les sciences de gestion (Detchessahar, 2019) étudient l'alliance entre la performance et la santé depuis de nombreuses années. Aussi, lorsque nous parlons de démarche QVT, c'est bien en nous référant aux travaux scientifiques menés autour de cette question-là. Combiner performance et santé exige en effet de parler de qualité du travail et de développement du pouvoir d'agir plutôt que de parler de qualité de vie au travail (Clot, 2021a). Si l'on souhaite aboutir à une "performance réelle» (Clot, 2021b), le dialogue autour de la performance doit cesser d'être réservé aux seuls dirigeants pour s'étendre aux managers, aux opérateurs ou encore aux bénéficiaires du travail (clients ou usagers). La méthodologie doit ainsi agir "sur les processus à l'origine de la configuration de la situation de travail » (Coutarel, Caroly, Vézina, \& Daniellou, 2015, p. 19). De manière concomitante, le dialogue institutionnel sur la santé au travail doit migrer de discussions généralement centrées sur des causes individuelles et/ou la gestion des risques vers des discussions centrées sur le travail et l'organisation du travail. Ce mouvement exige en lui-même une transformation des représentations individuelles, collectives et institutionnelles sur la santé, le travail et leurs liens respectifs. Certains auteurs parlent d'une progression du niveau de maturité qu'ils définissent comme «le rapport que l'organisation entretient avec les liens qui s'établissent entre le travail et la santé, influencé par des événements divers (d'ordre juridique, médiatique, social, organisationnel, individuel), qui agissent sur sa prise de conscience du rôle que joue le travail dans la construction et la dégradation de la santé et qui soutient une approche vers un questionnement institutionnalisé des questions de santé au travail » (Rouat \& Sarnin, 2018, p. 343). En ce sens, la qualité d'une démarche QVT et la durabilité des transformations générées reposent sur sa capacité à développer des processus de coopération et de maturité de manière interactive et concomitante aux niveaux individuel, collectif et organisationnel autour des questions de santé et de performance. C'est ainsi qu'elle parviendra à faire émerger le réel du travail au sein d'espaces de controverses ouverts entre les acteurs (Clot, 2014) pour construire des 
« compromis d'action et de règles locales produisant des effets à différents niveaux de sens (personnel, collectif, organisationnel)» (Detchessahar, 2019). Aboutir à ces transformations de manière durable (Brun, Biron, \& St-Hilaire, 2007) nécessite la coopération d'un maximum d'acteurs internes, notamment des dirigeants supérieurs (Coutarel \& al., ibid ; Guidou, 2019). L'intervention peut en effet se doter d'un caractère pédagogique si elle associe les acteurs au processus d'intervention (Dugué et al., 2010). Cela permet de renforcer ou de démontrer son utilité sociale (Rouat \& Sarnin, 2013) et ce faisant, d'internaliser l'intentionnalité. Il a également été montré que la démarche doit porter attention aux éléments contextuels pour s'intégrer à la vie de l'entreprise (Biron, Karanika-Murray, \& Cooper, 2012 ; Hansez, Bertrand, \& Barbier, 2009) et s'articuler à ses projets réels et à sa planification stratégique (Biron, St-Hilaire, BarilGingras, Paradis, Chabot, Lefebvre et al., 2016 ; Valléry \& Leduc, 2012). À la lecture de ce rappel de quelques-uns des déterminants de la transformation, on comprend que transformer durablement les situations de travail et l'organisation pour plus de santé et de performance, renvoie à un processus social fort complexe appelant de multiples transformations situées à différents niveaux, s'inscrivant dans différents temps, se jouant dans différents espaces, touchant à des objets aux ontologies diverses et transformant les sujets (Guidou, 2017). Cette complexité engendre une diversité d'obstacles à dépasser, non seulement pour les sciences du travail et pour les intervenants certes, mais aussi pour les acteurs des milieux de travail. C'est sur ce dernier point que nous souhaitons insister.

3 En matière de démarche de prévention de la santé psychique, qu'elle soit nommée de QVT ou d'un de ses thèmes associés (de bien-être au travail, de prévention des risques psychosociaux, du stress...), il existe une grande variété de méthodes (Valléry \& Leduc, ibid). Les entreprises peuvent donc se voir proposer des modèles d'intervention très divers, voire contradictoires. La carence des travaux d'évaluation ne facilite pas leur choix puisqu'il n'existe pas d'évidence scientifique concernant leur efficacité (Rouat \& Sarnin, 2018). Par ailleurs, les acteurs internes ont souvent grande peine à se représenter tout ce qui sera en jeu au cours de la démarche. Ce point est souvent attribué à un manque de connaissances des acteurs sur lesdites démarches. Cela est possible, mais nous semble partiel puisque l'objet même du travail à réaliser est instable. Selon les représentations, mais aussi selon leurs évolutions au gré des processus de maturation et de coopération, l'objet de travail migrera, souvent de la mauvaise santé (avec l'ensemble des indicateurs et artefacts créés pour l'évaluer) au travail, une notion qui se chargera progressivement des connaissances et méthodes utilisées par les sciences du travail. Cette instabilité de l'objet de travail se double d'une instabilité des destinataires de la démarche (Clot, 2006) : l'adressage pourra circuler des salariés, aux managers, aux instances du personnel, aux dirigeants ou encore aux institutions réglementaires. On notera également que les acteurs y sont souvent amenés à reconsidérer des actions passées (Sarnin, Durif-Bruckert, \& Rouat, 2011) ; ce qui équivaut finalement à travailler pour défaire le travail fait par ailleurs. Les obstacles ne relèvent donc pas seulement d'une complexité que nous pourrions qualifier de cognitive, mais se chargent aussi d'une autre nature qui peut être qualifiée d'affective (Clot, 2018). L'affect est une manifestation de l'émotion qui se forme devant le différentiel «entre nos attendus et les inattendus de l'activité en cours. [Lorsqu'il survient] un travail de révision est nécessaire [et] nous affecte en faisant osciller les rapports de forces dans la marche du connu vers l'inconnu " (Bonnefond \& Clot, 2016, p. 4). Ainsi, il apparaît qu'une telle démarche entraîne une perturbation des sujets au 
sens de Devereux (1967). Une absence de soutien face à cette perturbation participe donc de la difficulté à passer à l'action transformatrice. Pour le dire autrement, soutenir l'action transformatrice dans un milieu de travail exige également de soutenir les acteurs internes de ce milieu aux prises avec ces transformations.

\section{Proposition d'un dispositif d'innovation transitionnelle (DIT)}

4 Le DIT a été élaboré afin d'articuler un processus d'innovation organisationnelle (West \& Far, 1900) avec un processus transitionnel (Winnicott, 2002/1953). Nous émettons l'hypothèse que cette combinaison est susceptible de répondre aux finalités d'une démarche QVT, de dépasser les limites de la transformation durable, mais aussi de soutenir les acteurs internes face aux obstacles à traverser. Expliquons tout d'abord le lien fait entre démarche QVT et innovation organisationnelle. Il apparait premièrement au niveau du sens. Une démarche QVT tente de combiner santé et performance. Cette dernière amène notamment les entreprises à rechercher plus d'innovations, mais aussi à faire évoluer leur organisation (Detchessahar, 2019; Ughetto, 2018). Or, puisque la réussite d'un processus de transformation nécessite d'articuler la démarche à la vie et à la stratégie de l'entreprise et que l'implication des acteurs repose notamment sur l'utilité sociale de la démarche, la proposition d'un dispositif d'innovation organisationnelle semble constituer un objet d'intervention suffisamment global pour combiner tous les enjeux (Guidou, 2017). Secondairement, le lien entre QVT et innovation organisationnelle apparaît dans la proximité entre les processus recherchés. En effet, il est possible de dire qu'une démarche QVT est une « introduction volontaire et [une] mise en œuvre, dans un groupe et une organisation, d'idées et de processus innovants importants pour l'unité concernée afin d'apporter des bénéfices significatifs aux individus, aux groupes, à l'organisation et à la société ». Or, il s'agit de l'exacte définition de l'innovation selon West \& Far (ibid). De plus, puisque l'intervention vise la transformation effective et durable, il est correct de qualifier ses transformations d'innovations, au sens d'idées qui se sont diffusées (Moatty \& Tiffon, 2014). Enfin, notons en troisième point que le processus d'innovation est lié à celui de la créativité, cette dernière générant les idées qui pourront se transformer en innovation (Battistelli, 2014). Cette liaison entre innovation et créativité donne à l'innovation un potentiel de développement de la santé à condition qu'elle soit combinée à un mouvement d'appropriation de l'acte source de transformation de l'environnement (Amado, 2007 ; Prades, 2012).

5 Si le processus d'innovation organisationnelle présente des intérêts, il ne permet pas d'emblée d'accompagner les acteurs dans les obstacles de l'intervention. C'est pour y répondre que nous l'avons fait reposer sur un processus transitionnel. Durant ses travaux, Winnicott a étudié «le mouvement qui conduit de la relation aux objets subjectivement fermée sur elle-même jusqu'au domaine de l'utilisation de l'objet ${ }^{1} »(2000 / 1969$, p. 234). Il crée le concept d'objet transitionnel (OT) pour étudier le mouvement de l'un à l'autre. La confrontation au réel oblige en effet « à [affronter les] écarts dramatiques entre ce qui est donné et ce qu'on attend» (Reille-Baudrin, 2011, p. 47). Pour que cette confrontation aboutisse à la capacité de faire usage des objets du monde, un espace transitionnel est nécessaire. En son sein, si l'OT est reconnu socialement et permet un partage des représentations, il offre alors une alliance des 
expériences illusoires : lorsque tous partagent l'idée (on pourrait dire l'illusion) que tel projet permettra d'obtenir tel résultat, ils partagent une même combinaison (illusoire encore à ce stade) entre ce qu'ils désirent et ce qui est réel. Cette expérience partagée ouvre la voie à une activité particulière, nommée activité transitionnelle, au travers de laquelle le sujet transforme l'objet trouvé dans l'environnement et donné par le social (c'est-à-dire le projet), en objet (projet) créé. L'OT, grâce à ses caractéristiques et sa mise travail au sein de l'espace transitionnel, résiste aux attaques et supporte toute une palette d'affects allant de l'agressivité à la contribution et à la sollicitude (Winnicott, 1999/1948). Ainsi, l'usage de l'OT transforme le sujet. Cette activité renvoie à une créativité située, mobilisant autant le corps que la pensée et se manifestant dès l'agir : "l'impulsion peut être au repos, mais si l'on emploie le mot "faire", c'est qu'il y a déjà créativité" (Winnicott, ibid, p.43). La créativité renvoie ainsi à une volonté de " chercher à atteindre »; cette même créativité nécessaire à l'innovation et à la santé. L'hypothèse suivante peut donc être formulée: un dispositif d'innovation organisationnelle reposant sur un processus transitionnel est susceptible d'atteindre les finalités d'une démarche QVT tout en dépassant les limites de la transformation durable et en soutenant les acteurs. Le processus d'innovation organisationnelle doit pour ce faire, reposer sur la création d'un espace transitionnel, dans lequel les acteurs peuvent se saisir d'un objet donné par le milieu (ici par l'entreprise), et agir sur lui de manière créative afin de construire un objet-créé (projet-créé), cet objet permettant de supporter le «drame ordinaire de la vie quotidienne » (Winnicott, 2002/1953, p. 48) ; et donc potentiellement, de supporter les épreuves du dispositif d'intervention.

\section{Méthodologie}

\subsection{Contexte}

Le DIT a été élaboré dans le cadre d'une thèse de doctorat réalisé au sein d'un service de santé au travail (Guidou, 2017). Son expérimentation a été possible sous la forme d'une recherche-action proposée à un adhérent particulier du service de santé (SST). Il s'agit de l'une des quatre antennes d'un institut régional de formation supérieure pour lequel le médecin du travail avait alerté officiellement de situations de souffrance au travail dès 2014. Le site comprend 17 formateurs permanents, des formateurs vacataires et 8 employés de fonctions transversales (secrétaire, documentaliste...). Sa gouvernance est assurée par un conseil d'administration, un directeur général (DG), un secrétaire général (SG) et enfin un directeur local (dir.). Diverses actions avaient été menées par l'entreprise sans améliorer la situation. Une nouvelle alerte avait été faite en 2015. Les dirigeants ainsi que les élus du personnel ont alors sollicité leur SST. Leur demande urgente - portait à la fois sur la nécessité de «faire cesser les violences internes », de « retrouver un bien-vivre ensemble au travail », mais aussi « de résoudre durablement et sérieusement les conflits qui opposaient formateurs et direction, notamment quant à la surcharge de travail». L'ensemble des professionnels était fortement éprouvé et exprimait le sentiment "de ne plus savoir comment s'y prendre» (y compris le médecin du travail). C'est dans ce contexte que le DIT a été présenté (aux dirigeants puis aux élus du personnel et enfin à l'ensemble des salariés). Malgré sa validation en juillet $2016^{2}$, une grève a été déclenchée et a abouti au remerciement du DG par le conseil d'administration en septembre. Le directeur du site, placé en arrêt maladie, ne reprit jamais ses fonctions. Un nouveau DG, également directeur du site concerné, fut 
nommé en octobre lors du lancement du dispositif. Le DIT a ainsi été expérimenté dans un contexte très dégradé. Si sa mise à l'épreuve est donc importante, les SST observent souvent un tel niveau de dégradation. Cela correspond donc à un déploiement en situation réelle.

\subsection{Méthode}

7 La première étape du DIT consiste en la conduite de groupes de génération d'idées. Ces ateliers visent la production d'idées par les formateurs $(\mathrm{N} 1=17)$ d'une part et par les managers $(\mathrm{N} 2=3)$ d'autre part. Leur finalité est de débuter l'implantation du processus d'innovation émergente (Battistelli, 2012), d'ancrer les processus de créativité dans le réel, mais aussi de poser les bases d'une activité transitionnelle. Les participants y sont invités à imaginer des idées pour transformer leur situation de travail en " partageant une occasion d'imaginer d'autres possibles de [leur] activité, en énonçant des idées nouvelles, en explorant des pistes de transformation, même des pistes qui [leur] semblent a priori incongrues ou impossibles » (extrait de l'animation). Après l'élection d'une idée, le groupe "réfléchit à la forme qu'elle pourrait prendre dans [leur] quotidien de travail ». Au fil des séances, les «formes d'idées » se stabilisent et les moyens nécessaires à leur appropriation sont abordés. Ces idées ne sont pas produites ex-nihilo mais sont trouvées dans l'environnement de travail et héritées d'une histoire collective partagée. Au fil de cette "mise en forme collective", elles permettent une première confrontation entre ce que les participants désirent et ce qui existe ( $\mathrm{y}$ compris les différences de points de vue). Les idées-trouvées deviennent des projetscréés et acquièrent une valeur pour la communauté.

8 La deuxième étape du DIT correspond au regroupement du comité de pilotage (COPIL). Sa constitution fait l'objet d'une grande attention. Plusieurs conditions sont posées lors de la présentation du dispositif : tout d'abord, le COPIL est une instance représentative de l'établissement. Il comprend donc des représentants de la direction supérieure, de la direction locale ${ }^{3}$, des élus du personnel et des salariés (appel à volontariat pour ces derniers ${ }^{4}$ ). Ensuite, le COPIL est présenté comme l'instance décisionnelle de la démarche, combinant espaces de discussion et de décision (Habermas, 1987). Au cours de leur première journée de travail, les membres du COPIL prennent connaissance des projets-créés, débattent de leur pertinence, élisent ceux qu'ils souhaitent expérimenter, en font évoluer la forme puis identifient les activités qui seront transformées. L'animation est structurée en plusieurs étapes. Dans un premier temps, les objectifs du COPIL sont rappelés, à savoir contribuer à la maturation de projets devant transformer durablement les situations de travail. On insiste sur le fait que chaque membre porte la responsabilité de cette dynamique, afin de construire progressivement les conditions identifiées par Winnicott pour obtenir un objet transitionnel. En effet, les projets réfléchis dans cet espace-temps n'appartiennent pas totalement à la réalité extérieure (ils n'existent pas encore dans le quotidien), mais ne sont pas non plus totalement intérieurs (ils sont discutés entre acteurs et visent à se réaliser). Ils se situent dans un espace intermédiaire. Ensuite, en confiant la responsabilité des projets au COPIL, le DIT agit sur leur valeur symbolique et en développe une reconnaissance sociale partagée. Le second temps d'animation vise la création d'illusions partagées entre les membres malgré leur fonction différente au sein de l'organisation. Si le terme «illusion » appartient au vocabulaire psychanalytique, il est possible de le conserver car les projets ou idées de transformation sont partiels tant 
qu'ils n'ont pu se confronter au travail réel et aux différences de points de vue. C'est en effet au travers de leur confrontation sur la base de l'analyse de l'activité qu'il sera possible d'aboutir à des transformations articulant santé et performance. En ce sens, il est correct de dire que le DIT ne crée pas d'illusion, mais prend acte de ces idées ou projets «illusoires» qui existent. Il cherche tout d'abord à passer d'illusions individuelles ou spécifiques à un groupe particulier, à des illusions collectives partagées pour ensuite accompagner le passage de l'illusion au réel (chez Winnicott, de la relation à l'objet à l'utilisation de l'objet). Pour construire ces illusions partagées, les projetscréés lors des groupes de génération d'idées ne sont pas présentés immédiatement. Au préalable, c'est une formulation simple de leurs orientations qui est proposée et mise en débat. Cette formulation rend compte de ce que les projets-créés «cherchent à atteindre ", c'est-à-dire de l'impulsion créative. Elle débute donc par "aller vers ». Après avoir pris connaissance des orientations des projets-créés au sein des groupes de génération d'idées des salariés et des managers, le COPIL est invité à en débattre pour construire des illusions partagées: «Le premier temps du COPIL doit être celui d'un accord de ses membres sur ces orientations afin de s'assurer que vous partagez tous ces objectifs à atteindre» (extrait de l'animation). Par exemple, dans cette expérimentation, les formulations partagées ont été : 1) «aller vers un engagement individuel et collectif pour le mieux et bien vivre-ensemble », 2) «aller vers des temps collectifs entre formateurs pour échanger sur les pratiques ", «3) «aller vers un cadre interétablissements permettant l'adaptation aux réalités locales»,4) "aller vers une valorisation, un partage et une capitalisation des ressources» et 5) «aller vers une programmation et un plan de charge qui remettent le travail au centre ». Le COPIL est ensuite invité à identifier des indicateurs d'évaluation de ces transformations désirées. Par exemple, quels indicateurs permettraient de mesurer que les projets ont bien permis « d'aller vers un engagement individuel et collectif pour le mieux et bien vivreensemble »? Le partage des illusions est ainsi renforcé par un travail d'alliance entre les désirés et le réel et l'ancrage des projets dans le réel est soutenu (Battistelli \& Picci, 2008). Lors de la troisième étape d'animation, les projets des groupes de génération d'idées sont présentés : le COPIL est alors en situation de trouver des projets qui leur sont donnés par d'autres, qui les ont eux-mêmes trouvés dans leur environnement. Après avoir pris connaissance de leur forme, le COPIL sélectionne ou plutôt élit le ou les projets qu'il souhaite développer. Au sein de cet institut, trois projets ont été retenus. Lors de la quatrième séquence d'animation, le COPIL réfléchit aux formes que pourraient prendre ces projets à partir de celles proposées, transformant ainsi ces projets-donnés en projets-créés et leur conférant les caractéristiques d'objets/projets transitionnels. Enfin, la cinquième étape d'animation vise à ancrer ces projets dans le réel. Cette liaison est essentielle pour soutenir la transformation et le processus transitionnel. En effet, c'est bien la confrontation de l'OT au réel qui permet d'engendrer l'activité transitionnelle. Ainsi, le COPIL est invité à identifier les activités susceptibles d'être transformées par les projets. Durant l'ensemble de cette journée de travail, l'intervenant propose les différentes séquences et anime les échanges en assurant le cadre, en veillant à l'expression de tous, en reformulant les propos ou encore en mettant en exergue les points d'accord et de désaccord, mais n'intervient pas dans les débats : il ne donne pas son point de vue sur les orientations, sur les formes des projets ni même sur les indicateurs proposés. Son rôle est de proposer et de maintenir le cadre au sein duquel les membres du COPIL élaborent et débattent, ce cadre étant 
construit pour générer l'activité transitionnelle qui par elle-même transforme les projets et les acteurs.

9 La troisième étape du DIT consiste à étudier les activités potentiellement transformées par les projets. Il s'agit d'une étape plus ou moins longue selon les projets. Ici, l'intervenant est maître des méthodes afin de réaliser une analyse qualitative du réel de l'activité (Amado \& Lhuillier, 2012 ; Sarnin, Caroly, \& Douillet, 2011), permettant d'aller au-delà des tâches et des représentations. Comme on l'a vu, il est en effet nécessaire de confronter les acteurs au réel. Quatre spécificités sont à relever : d'une part les activités analysées se situent dans le périmètre des projets transitionnels construits dans le COPIL. D'autre part, l'analyse implique le plus possible de salariés (employés comme managers), l'implication renvoyant autant à la participation du plus grand nombre qu'à leur positionnement dans un rôle actif (Guidou, 2017). Il s'agit toujours de renforcer l'ancrage et l'appropriation des projets par le collectif, ainsi que leur caractère émergent. De plus, du point de vue du processus transitionnel, cette large implication permet aux acteurs qui avaient participé aux groupes de génération d'idées, de retrouver les projets qu'ils avaient donnés au COPIL, de prendre connaissance des projets qui ont été créés, mais aussi de les faire évoluer au gré des étapes d'analyse de l'activité. Pour implanter ce processus et développer les compétences des acteurs de l'entreprise, des salariés nommés « analystes du travail » sont impliqués sur la base du volontariat ${ }^{5}$ $(\mathrm{N}=5)$ et bénéficient d'une formation-action ${ }^{6}$. Les méthodes d'analyse de l'activité sont adaptées à la nature des projets. Par exemple le $3^{\mathrm{e}}$ projet retenu par le COPIL visait l'instauration de séances d'analyses de la pratique. Il répondait à la fois au «besoin de verbalisation et d'élaboration autour des vécus de souffrance et au besoin d'échanges sur le travail ». L'analyse devait donc permettre d'identifier les besoins des salariés quant aux épreuves du travail, quant aux ressources dont ils disposent ou dont ils souhaitent bénéficier via ce nouvel espace, mais aussi d'identifier les articulations à penser entre celui-ci et les autres niveaux de régulation interne. Au regard de ces enjeux, la méthodologie issue de l'enquête en psychodynamique du travail a été utilisée (Dejours \& Bègue, 2009). Elle permet d'aboutir à une étiologie de la crise et développe de nouvelles compétences collectives en instaurant des espaces de délibération et en transformant le rapport subjectif au travail en "(déclenchant) la pensée par la pensée" (Clot \& Leplat, 2005, p. 18). D'autre part, son objectif porte sur la transformation effective de l'organisation du travail en amenant les salariés à analyser leurs vécus au regard de cette organisation (Dejours \& Bègue, ibid). Les analyses du travail de ce projet ont donc consisté à animer trois séances collectives auprès des formateurs volontaires via cette méthodologie afin d'en extraire les besoins à formuler concernant l'instauration d'analyses de pratiques. Le premier projet visait à « créer un modèle de management posant un cadre interétablissements et permettant l'adaptation aux réalités locales » et le second à " restructurer les espaces collectifs ». Pour étudier ces deux projets, les analyses ont été regroupées et ont consisté en des séances collectives animées selon la méthodologie de la médiation par l'activité proposée par la psychosociologie du travail (Amado et Lhuilier, 2012). Enfin, le quatrième projet, qui portait sur la création d'un parcours d'intégration des formateurs, a donné lieu à des entretiens individuels réalisés via une grille d'entretien semi-directive auprès de formateurs récemment arrivés dans l'institut d'une part ainsi qu'à des instructions au sosie (Clot, 2017) auprès des formateurs plus anciens afin d'identifier leurs pratiques de transmission du métier. Pour chacun des projets, les acteurs impliqués étaient systématiquement invités à faire évoluer la forme des projets-créés par le COPIL à la 
lumière des analyses de l'activité. Ce faisant, les projets se chargent progressivement de l'ensemble des points de vue et de la complexité de l'activité tandis que l'activité transitionnelle est élargie à l'ensemble des salariés (salariés et managers).

La quatrième étape du DIT regroupe à nouveau le COPIL pour présenter les analyses des activités ainsi que les nouvelles formes de projets proposés. En référence à la théorie winnicottienne, l'animation confronte les membres du COPIL aux activités réelles. Il s'agit d'animer une controverse professionnelle à partir de la révélation des écarts entre travail prescrit et réel, des critères de qualité de travail, des épreuves du travail et des vécus associés. Pour ce faire, les analyses sont présentées par l'intervenant et les analystes du travail. Le COPIL poursuit l'activité transitionnelle précisément parce que ses membres doivent combiner leurs illusions avec le réel ; réel ramené dans ses formes les plus éprouvantes via les analyses du travail. L'aire transitionnelle créée permet aux projets transitionnels de résister, le COPIL pouvant alors affronter «les écarts dramatiques entre ce qui est donné et ce qu'on attend» (Reille-Baudrin, 2011, p. 47). Progressivement, ils passent de la relation à l'activité (registre de l'expérience subjective) à son utilisation (registre de l'expérience vécue) et vont de l'agressivité à la contribution responsable. L'usage des OT, ici les projets d'innovation, transforme les acteurs. Ce COPIL s'achève avec la stabilisation des formes des projets (nouveaux projets-créés) puis par la validation de leurs expérimentations en situation réelle.

11 La cinquième étape du DIT consiste en l'expérimentation des projets innovants selon les modalités validées. L'élargissement des projets à l'ensemble des salariés, mais également leur mise à l'épreuve de la transformation réelle, permet une nouvelle confrontation. Leur capacité d'innovation est également évaluée en testant leur combinaison avec les pratiques antérieures (Mallein \& Toussaint, 1994).

La sixième étape clôture le DIT par une troisième journée de travail du COPIL. L'animation y est proche du second COPIL, les retours des expérimentations confrontant une nouvelle fois ses participants en leur permettant de poursuivre l'activité transitionnelle en stabilisant les nouvelles formes des projets. Le processus est finalisé en sécurisant le processus d'innovation : les objets-créés sont transformés en objets-frontières (Peters, Faulw, \& Hansez, 2010), c'est-à-dire en objets permettant de soutenir la communication entre ceux qui ont innové et ceux à qui l'on présente l'innovation (Star \& Griesemer, 1989). L'ensemble des étapes et des moyens de mise en œuvre des projets est défini. La question du «qui est responsable du déploiement » permet aussi d'identifier des "passeurs» internes (Alter, 2000) qui diffuseront les projets d'innovation en enrôlant les autres acteurs (Peters et al., ibid, p. 67). Enfin, le COPIL s'achève par la définition d'indicateurs de suivi des innovations et la programmation des dates de réunion de l'instance en charge. Il s'agit ici d'assurer l'étape de stabilisation ou routinisation des innovations (Battistelli, 2014).

L'ensemble des étapes (voir tableau $\mathrm{n}^{\circ}{ }^{1}$ ) du DIT est précisé ci-dessous en prenant soin de rappeler les acteurs mobilisés (VP: vice-président, SG : secrétaire général, DG : directeur général), le nombre, la durée des séances et leurs objectifs ainsi que les données recueillies pour évaluer les effets du dispositif. 
Tableau 1 : Synthèse du DIT.

Table 1 : Transitional Innovation Device (DIT) overview

\begin{tabular}{|c|c|c|c|c|}
\hline & $\begin{array}{c}\text { Acteurs } \\
\text { mobilisés }\end{array}$ & Séances & Objectifs & Données recueillies \\
\hline $\begin{array}{l}\text { Groupes de } \\
\text { génération d'idée } \\
\text { (oct. à déc. } 2016\end{array}$ & $\begin{array}{l}\text { Salariés } \\
\text { VP, SG, DG }\end{array}$ & $\begin{array}{l}7^{1} \text { groupes de } 2 \mathrm{~h} \\
3 \text { groupes de } 2 \mathrm{~h}\end{array}$ & $\begin{array}{l}\text { Co-générer des idées } \\
\text { pour améliorer les } \\
\text { situations de travail et } \\
\text { co-élaborer leur } \\
\text { ancrage dans le réel }\end{array}$ & $\begin{array}{l}\text { Enregistrement audio } \\
\text { Nombre d'idées émises } \\
\text { Nombre et forme des } \\
\text { idées retenues et des } \\
\text { projets élaborés }\end{array}$ \\
\hline $\begin{array}{l}\text { COPIL 1 } \\
\text { (janv. 2017) }\end{array}$ & $\begin{array}{l}4 \text { salariés } \\
\text { volontaires, } \\
\text { un salarié elu } \\
\text { du personne1, } \\
\text { VP, SG, DG }\end{array}$ & 1 journée & $\begin{array}{l}\text { Prendre connaissance } \\
\text { des projets, élire les } \\
\text { projets d'innovation, } \\
\text { identifier les activités à } \\
\text { analyser }\end{array}$ & $\begin{array}{l}\text { Enregistrement audio } \\
\text { Indicateurs construits par } \\
\text { le COPIL pour évaluer } \\
\text { les transformations } \\
\text { visées }\end{array}$ \\
\hline $\begin{array}{l}\text { Analyse des } \\
\text { activités } \\
\text { (fév. à mars 2017) }\end{array}$ & $\begin{array}{l}5 \text { analystes du } \\
\text { travail } \\
\text { Salariés } \\
\text { SG }\end{array}$ & $\begin{array}{l}\text { Projet } 1 \text { et } 2: \\
2 \times 3 \mathrm{~h} \\
\text { Projet } 3: \\
3 \times 2 \mathrm{~h} \\
\text { Projet } 4: 2 \\
\text { entretiens } \\
\text { individuels, } \\
3 \text { instructions au } \\
\text { sosie }\end{array}$ & $\begin{array}{l}\text { Participer aux analyses } \\
\text { de l'activité et co- } \\
\text { élaborer les projets } \\
\text { innovants }\end{array}$ & $\begin{array}{l}\text { Prises de note concernant } \\
\text { l'analyse des activités en } \\
\text { séances collectives } \\
\text { Enregistrement audio des } \\
\text { entretiens } \\
\text { Nombre et évolution des } \\
\text { formes des projets }\end{array}$ \\
\hline $\begin{array}{l}\text { COPIL 2 } \\
(\text { mars 2017) }\end{array}$ & $\begin{array}{l}4 \text { salariés } \\
\text { volontaires, } \\
\text { un salarié élu } \\
\text { du personnel, } \\
\text { VP, SG, DG }\end{array}$ & $\begin{array}{l}2 \text { demi-journées } \\
\text { de } 4 \mathrm{~h}\end{array}$ & $\begin{array}{l}\text { Prendre connaissance } \\
\text { des analyses de } \\
\text { 1'activité et des } \\
\text { nouvelles formes } \\
\text { proposées, confronter } \\
\text { les projets au réel, co- } \\
\text { transformer les projets, } \\
\text { prévoir les } \\
\text { expérimentations } \\
\end{array}$ & $\begin{array}{l}\text { Enregistrement audio } \\
\text { Nombre et évolution des } \\
\text { formes des projets } \\
\text { Indicateurs d'évaluation } \\
\text { construits par le COPIL }\end{array}$ \\
\hline $\begin{array}{l}\text { Expérimentation } \\
\text { (avril à juin 2017) }\end{array}$ & $\begin{array}{l}\text { Salariés, SG, } \\
\text { DG }\end{array}$ & & $\begin{array}{l}\text { Expérimenter les } \\
\text { projets dans le réel, } \\
\text { tester leur } \\
\text { combinaison avec les } \\
\text { pratiques }\end{array}$ & $\begin{array}{l}\text { Réunion conduite par } \\
\text { l'intervenant pour } \\
\text { évaluer le suivi des } \\
\text { indicateurs }\end{array}$ \\
\hline $\begin{array}{l}\text { COPIL 3 } \\
\text { (juin 2017) }\end{array}$ & $\begin{array}{l}4 \text { salariés } \\
\text { volontaires, } \\
\text { un salarié êlu } \\
\text { du personnel, } \\
\text { VP, SG, DG }\end{array}$ & 1 journée & $\begin{array}{l}\text { Prendre connaissance } \\
\text { des expérimentations, } \\
\text { décider des suites des } \\
\text { projets, co-élaborer } \\
\text { leur suivi }\end{array}$ & $\begin{array}{l}\text { Enregistrement audio } \\
\text { Nombre et évolution des } \\
\text { formes des projets }\end{array}$ \\
\hline
\end{tabular}

\section{Résultats}

\subsection{Les transformations obtenues et leur implantation}

14 L'innovation est un processus allant de la génération, à la promotion puis à la réalisation d'idées (Kanter, 1988). Elle suit un processus cyclique composé de périodes d'initiation, d'implémentation, d'adaptation et de stabilisation (Battistelli, 2014). Une innovation doit nécessairement être appropriée par ceux à qui elle est destinée (Alter, 1996), notamment en se greffant aux pratiques antérieures (Mallein \& Toussaint, 1994). Il faut donc distinguer innovation et invention : l'innovation n'a pas nécessairement de caractère de nouveauté et peut consister en l'appropriation de pratiques/produits qui existaient déjà, mais dans un autre milieu. Via le DIT, les innovations que nous cherchons à générer à partir d'un processus transitionnel, concernent l'organisation et les pratiques professionnelles. S'il n'est pas possible de détailler chacune d'entre elles dans cet article, nous pouvons rendre compte d'une question en particulier et de la manière dont le processus a permis son travail. Parmi les conflits existants entre formateurs et managers apparaissait particulièrement la question du plan de charge. Il s'agit d'un artefact construit sous la forme d'un tableur Excel recensant l'ensemble des formateurs et de leur temps de travail, des formations de chacune des filières et des années d'étude ainsi que les heures de "face à face pédagogique » et autres tâches liées aux tutorats ou aux stages des étudiants. À cette comptabilité avait été ajoutée, au fil des plaintes concernant la surcharge de travail, une quantification des temps de préparation jugés nécessaires pour chaque tâche. Au lancement du DIT, les échanges prenaient la forme d'une bataille de chiffres, les formateurs demandant plus d'heures 
de préparation, réduisant ainsi les temps de face à face et mettant les managers devant une impossibilité à programmer tous les cours. Les impasses, devenues fréquentes, se réglaient alors individuellement au gré des semaines et aboutissaient à ce que les formateurs appelaient "l'épicerie» du plan de charge. Voici l'évolution de cette question au fil du DIT (voir Tableau 2) :

Tableau 2 : Exemple de processus d'innovation reposant sur une activité transitionnelle. Table 2 : Example of an innovation process based on a transitional activity

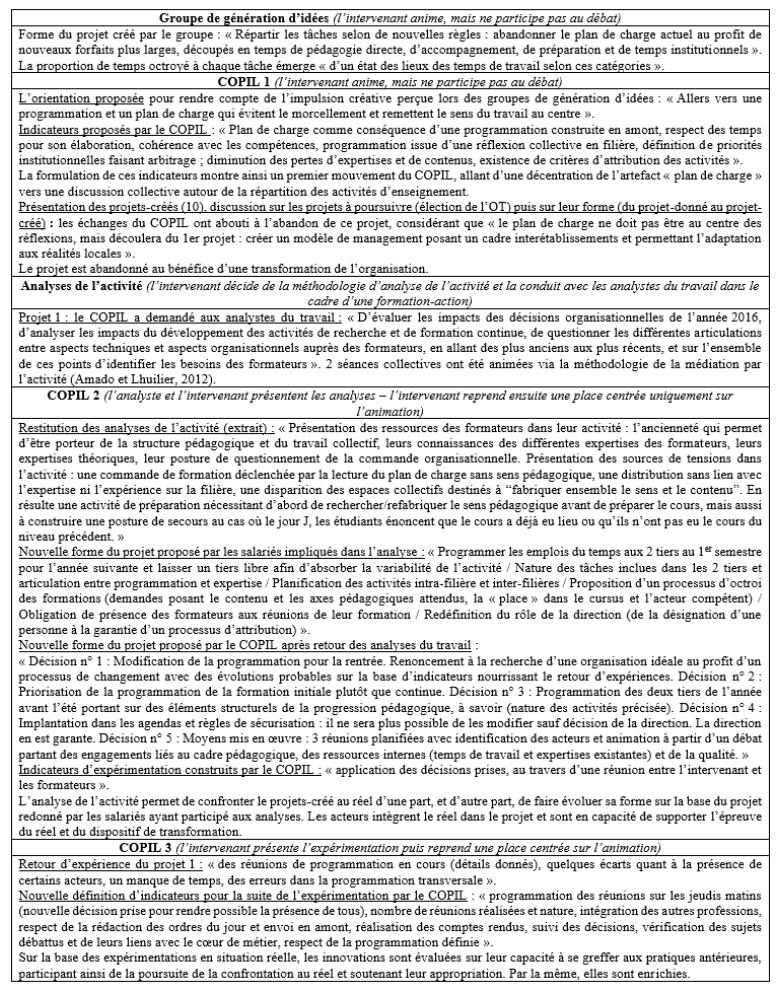

Le dispositif a permis d'observer d'autres évolutions à partir du même processus. Elles sont synthétisées ci-dessous (voir Tableau 3). 
Tableau 3 : Synthèse des transformations observées. Table 3: Summary of the observed transformations

\begin{tabular}{|c|c|}
\hline $\begin{array}{ll}\text { Grp } & \mathrm{de} \\
\text { générations } & \\
\text { d'idées } & \end{array}$ & 77 idées émises, 10 projets donnés au COPIL \\
\hline COPIL 1 & $\begin{array}{l}5 \text { orientations transmises par l'intervenant, } 42 \text { indicateurs formulés et } 5 \text { projets recréés par } \\
\text { le COPIL. 1) nouveau modèle de management, 2) restructuration des espaces collectifs, 3) } \\
\text { instauration d'analyse de la pratique (APP), 4) création d'un outil de ressource interne, 5) } \\
\text { création d'un parcours d'intégration des formateurs }\end{array}$ \\
\hline $\begin{array}{l}\text { Analyses } \\
\text { 1'activité }\end{array}$ & $\begin{array}{l}\text { Projet } 1 \text { et } 2: 2 \text { séances collectives - objectifs donnés aux analyses : voir tableau } 2 \\
\text { Projet } 3: 3 \text { séances collectives - objectifs donnés aux analyses : « cerner les attentes en vue } \\
\text { de la rédaction d'un cahier des charges permettant de sélectionner un intervenant » } \\
\text { Projet } 4: \text { abandon - absence de temps et d'analystes disponibles } \\
\text { Projet } 5: 2 \text { entretiens semi-directifs et } 3 \text { instructions au sosie - objectifs donnés aux } \\
\text { analyses : « s'entretenir avec les formateurs et stagiaires arrivés cette année et étudier leur } \\
\text { intégration, rencontrer les formateurs pour cerner les pratiques d'intégration, étudier les } \\
\text { documents et pratiques RH liés à l'arrivée de nouveaux formateurs » }\end{array}$ \\
\hline COPIL 2 & $\begin{array}{l}\text { Retour des analyses de l'activité, évolution des projets : } \\
\text { Projet } 1 \text { : voir tableau } 2 \\
\text { Projet } 2 \text { : Identification de l'ensemble des espaces de discussion et planification sur toute } \\
\text { 1'année, définition de ce qui s'y discute et selon quelle animation, des périmètres } \\
\text { décisionnels et des modalités de discussion intergroupes et avec la hiérarchie. } \\
\text { Projet } 3 \text { : APP accessiblea à tous les formateurs permanents comme occasionnels, accès sur } \\
\text { volontariat sauf pour la 1re année d'emploi où elle est obligatoire, définition des questions } \\
\text { qui pourront s'y travailler, modalité de composition des groupes, rythme d'une fois par mois } \\
\text { Projet } 5 \text { : Définition de l'ensemble des étapes d'intégration tel qu'un entretien avec le DG, } \\
\text { le tuteur (nouvelle fonction créée) et le SG en charge des RH, des entretiens de suivi avec le } \\
\text { tuteur puis un nouvel entretien tripartite en fin de période d'essai. Définition d'un processus } \\
\text { de montée en compétences. Formulation d'interdits, par exemple l'octroi d'activités de } \\
\text { coordination pédagogique inter-filieieres la première année d'emploi } \\
\text { Décisions d'expérimentation }\end{array}$ \\
\hline Expérimentation & Modalités adaptées aux projets \\
\hline COPIL 3 & $\begin{array}{l}\text { Retour des expérimentations } \\
\text { Projet } 1 \text { : voir tableau } 2 \\
\text { Projet } 2 \text { : stabilisation du projet du COPIL } 2 \text { et nouvelles décisions : définition du nombre } \\
\text { d'heures de réunions par filière et implantation dans les agendas les jeudis matin, à un } \\
\text { rythme d'une par mois, animation par le référent de filière, forme du compte rendu et } \\
\text { animation débutant systematiquement par le suivi des décisions } \\
\text { Projet } 3 \text { : validation du projet du COPIL } 2 \text { aprés rédaction d'un cahier des charges, choix } \\
\text { d'un intervenant et lancement des APP en septembre } 2017 \\
\text { Projet } 5: \text { stabilisation du projet du COPIL } 2 \text { et nouvelles décisions : tuteur désigné pour un } \\
\text { an, définition des temps de tutorat et implantation dans les agendas, définition d'un parcours } \\
\text { de découverte les } 15 \text { premiers jours, d'un mois de travail avec le tuteur puis d'un mois et } \\
\text { demi en autonomie, définition des êtapes de validation de l'intégration à } 3 \text { mois, constitution } \\
\text { d'un guide RH et de ses composantes à remettre à l'arrivée. }\end{array}$ \\
\hline
\end{tabular}

Nous pouvons souligner le dynamisme du processus d'innovation au regard des transformations observées quant aux règles institutionnelles, organisationnelles ou encore des pratiques quotidiennes. Rappelons que l'intervention s'est déroulée sur 10 mois dans un contexte dégradé. Ces résultats sont d'autant plus intéressants qu'ils sont le fruit de l'élaboration collective des acteurs internes et en aucun cas de l'intervenant. Ainsi, ils montrent qu'au travers du DIT, les acteurs internes ont su passer des étapes de diagnostic (connaissance sur les situations de travail et l'organisation) à la transformation, et à la transformation durable. En effet, un an après la fin du dispositif, 21 des 31 innovations restent effectives.

\subsection{Le développement d'activités transitionnelles}

Pour vérifier que les transformations observées reposent sur la présence d'activités transitionnelles, nous avons défini des indicateurs à rechercher dans les interactions au sein du COPIL, notamment: la résistance de l'objet lors de la confrontation au réel (souligné dans le texte) ; la reconnaissance sociale de l'objet visible par la répétition de son utilisation et l'alliance des représentations (en italique); les agirs créatifs (en gras). Voici le résultat de cette analyse sur un extrait du $2^{\mathrm{d}}$ COPIL (voir Tableau 4) : 
Tableau 4 : Travail des objets donnés, extrait du $2^{\mathrm{d}}$ COPIL (souligné : confrontation au réel ; italique : alliance des représentations; gras : agir créatif).

Table 4 : Work of given objects, extracted from second steering committee (underlined: comparison with reality; italics: representations alliance; bold: creative acts)

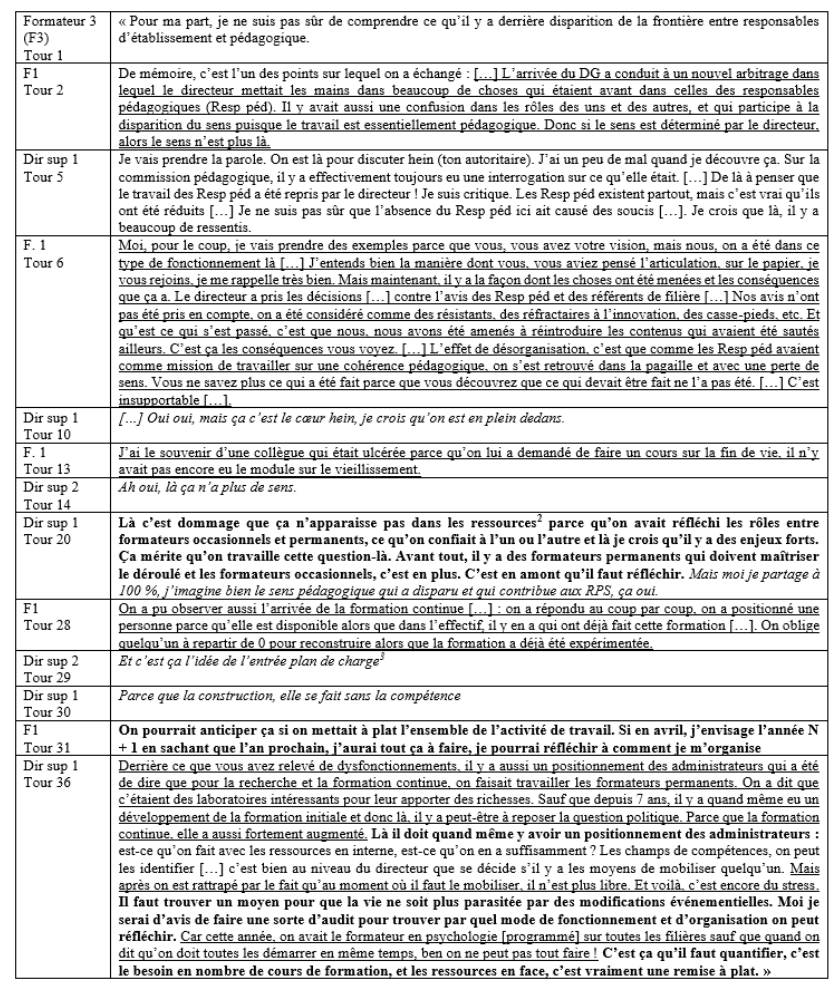

Avant la séquence retranscrite, les membres du COPIL étaient parvenus à partager un idéal de management au travers de ce qu'ils nommaient «une organisation d'intelligence collective ». Dans l'extrait, on voit (souligné dans le texte) l'ensemble des confrontations auxquelles cette illusion est soumise. Cette confrontation est issue du réel apporté par l'intermédiaire des analyses du travail. Particulièrement au tour 5 , on constate les émotions défensives d'un des dirigeants supérieurs qui voit sa représentation tomber (ce qui semble d'ailleurs presque reconnu par le formateur F. 1 lorsqu'il dit «j'entends bien la manière dont vous, vous aviez pensé l'articulation, sur le papier, je vous rejoins, je me rappelle très bien »). Pour autant, dès les tours 10,14 et 20 , on observe (en italique) que cette réalité est rapidement acceptée et aboutit à un agir créatif (en gras) : distinguer le rôle et l'activité des formateurs permanents de ceux des formateurs occasionnels. Cette capacité à supporter l'écart entre désiré et réel s'observe encore plus rapidement entre les tours 28 et 31 . Une confrontation du réel intervient (solliciter un collègue parce qu'il est libre alors qu'un autre a l'expérience), mais n'entraîne plus de réaction défensive. Et un agir créatif est bien observé : la mise à plat de l'ensemble des activités. Rappelons ici qu'il ne s'agit pas d'évaluer sa pertinence au regard des savoirs acquis par les sciences du travail, mais bien d'observer les impulsions créatives qui selon Winnicott (1999/1948) sont présentes dès qu'on emploie le mot « faire ", lequel signifie une volonté de « chercher à atteindre ». Au tour 36, cette capacité à faire face à l'écart entre désiré et réel semble être appropriée par l'un des dirigeants supérieurs : il apporte de lui-même de nouveaux écarts (la décision politique d'impliquer les formateurs permanents dans la formation continue et la recherche tandis que les charges augmentaient). L'ensemble de la séquence se conclut par un agir 
créatif: la réalisation d'un état des lieux permettant d'identifier l'ensemble des contenus et activités à réaliser d'une part et les ressources mobilisables d'autre part. Les phénomènes observés dans cet extrait ont été retrouvés dans l'ensemble des regroupements du COPIL. Au fil du dispositif, ses membres ont transformé les projets, devenus des projets transitionnels. Par l'intermédiaire de leur confrontation au réel apporté par les analyses de l'activité ; les acteurs ont été en mesure de supporter les épreuves affectives engendrées par l'intervention. Progressivement, des agirs créatifs ont été observés et l'ancrage des projets dans l'environnement s'est renforcé. Ces mouvements nous semblent donc à l'origine des transformations obtenues par le DIT.

\section{Discussion}

\subsection{Un développement de la QVT}

Cet article nous a permis de présenter une nouvelle méthodologie d'intervention construite pour répondre aux finalités d'une démarche QVT, dépasser les limites de la transformation durable, mais aussi soutenir les acteurs internes face aux obstacles à traverser. Dans le cadre d'une recherche-action conduite en SST, le DIT a été expérimenté pour la première fois dans un institut de formation supérieure dans un contexte particulièrement dégradé. Trois types de bénéfices ont été observés. Tout d'abord, l'organisation du travail, certaines pratiques et règles institutionnelles ont été transformées, et ce, de manière durable (un an après la clôture). Ensuite, des activités transitionnelles ont été observées entre les membres du COPIL, cette instance étant représentative et comprenant des salariés, des dirigeants et des représentants du personnel. Ces derniers ont mis au travail des projets innovants et les ont confrontés à l'activité et à l'expérimentation. Enfin, nous avons également observé une nette réduction des indicateurs d'absentéisme, de l'ordre de - $48 \%$ entre l'année de lancement du DIT et l'année scolaire suivante (- 410,5 jours d'arrêt entre octobre 2016 et juin 2018).

Ces bénéfices s'expliquent tout d'abord par la capacité du DIT à impliquer l'ensemble des acteurs tout au long du dispositif. Cela est aussi vrai pour les dirigeants supérieurs qui sont impliqués tout le long, et pas uniquement au début et à la fin. Il faut également relever que cette implication ne signifie pas seulement participation, mais aussi positionnement dans un rôle actif: ce sont les acteurs qui imaginent, débattent, élaborent, confrontent, et finalement innovent. Le second point essentiel réside dans la centration du dispositif sur le travail dès son commencement. En effet, puisqu'il vise l'innovation, le DIT ouvre des espaces de dialogues directement centrés sur le travail et sa transformation. La difficulté à passer d'un débat sur la santé à un débat sur le travail est ainsi réduite. L'utilité sociale de la démarche apparaît plus facilement (Rouat \& Sarnin, 2013), tout comme son intégration à la planification stratégique (Biron et al., 2016). Enfin, en confrontant les projets à l'analyse du travail et à l'expérimentation, la méthodologie permet bien une centration sur le travail réel (Cox, Taris, \& Nielsen, 2010). Le troisième point central repose sur le processus transitionnel créé. Ce dernier permet de soutenir les acteurs dans les épreuves affectives à traverser en proposant un cadre qui accompagne le «travail de révision [qui] nous affecte devant le différentiel entre nos attendus et les inattendus de l'activité en cours " (Bonnefond \& Clot, 2016, p. 4). De ce point de vue, on pourrait dire que le DIT permet de développer de manière 
concomitante les processus de maturité et de coopération autour du travail et de sa transformation.

\subsection{Un potentiel de transformation à ré-expérimenter}

21 Si les résultats obtenus sont encourageants, ils invitent à poursuivre l'expérimentation du dispositif, notamment pour mesurer l'impact du contexte d'intervention sur les effets observés. En effet, malgré la dégradation du contexte, la population était constituée de formateurs disposant d'un haut niveau de formation et d'une culture professionnelle favorable au questionnement et à la controverse. De plus, s'agissant de formateurs de futurs professionnels, ils avaient l'habitude de réfléchir au métier, à la construction des pratiques ou à leurs transmissions. Ces caractéristiques peuvent créer un environnement favorable aux processus de créativité. En effet, Amado (2015) rappelle que certains contextes facilitent ou entravent la créativité individuelle et collective. La "débrouillardise " selon ses termes, serait liée à des pratiques sociales permettant la reconnaissance et le contournement, si ce n'est la transgression des règles et des normes. Or, les analyses du travail ont effectivement fait apparaitre des compétences telles que la ruse, la capacité à changer de posture ou à anticiper des stratégies de secours. De même, même si le turnover de la direction a créé une grande instabilité au lancement du dispositif, l'arrivée d'un nouveau DG a sans doute permis de limiter les résistances à remettre en question des décisions passées (ce n'était toutefois pas le cas du SG ni du VP). Enfin, le contexte d'intervention est aussi celui d'un établissement de petite taille. Or, puisque le DIT insiste particulièrement sur la participation du plus grand nombre, on peut se demander quelles seraient les modalités à imaginer dans une entreprise de grande taille.

Lors d'une nouvelle expérimentation du dispositif, il nous apparaît également nécessaire d'analyser plus précisément comment la controverse professionnelle "se joue ", notamment lors des passages entre les différents espaces collectifs du DIT (entre les groupes de génération d'idées et le COPIL 1 par exemple). Il faudrait également analyser si et comment l'activité transitionnelle s'élargit au sein de l'organisation et des groupes professionnels ou encore s'il n'existe pas un risque de voir "neutraliser " la dispute professionnelle par l'activité transitionnelle. Par exemple, au sein de cet institut de formation, il serait intéressant de revenir sur site à distance afin de comprendre comment les transformations ont évolué et comment les différences de points de vue continuent de se discuter. En ce sens, l'évolution de la fonction « analyste du travail» nouvellement créée pourrait constituer un objet d'étude intéressant, notamment pour vérifier que les acteurs continuent de débattre à partir de l'activité.

\subsection{Vers une liaison entre créativité, innovation et santé}

Le DIT permet une alliance entre créativité, innovation et santé. En effet, si l'innovation est généralement perçue par les entreprises comme source de progrès, elle n'en est pas moins un processus complexe. Puisqu'elle "transgresse les conventions du monde » (Alter, 2000, p. 28), l'innovation engendre toujours des tensions chez ceux à qui elle s'adresse (résistance externe), mais aussi au sein du collectif qui la génère (résistance interne). Elle ne constitue pas nécessairement une avancée pour la santé et la qualité du travail et peut même être source de mal-être si elle est bâtie en dehors du réel du travail, se construit sur des a priori et sans dialogue social (Giusto, 2014), empêche la 
réalisation du beau travail ou encore se traduit par des objectifs inatteignables et une intensification du travail (Davezies, 2014; Dejours, 1998; Goulet \& Vinck, 2012). En fondant le processus d'innovation sur un processus transitionnel comprenant en luimême une confrontation au réel, le DIT semble permettre le dépassement de ces limites. Sa méthodologie accompagne les tensions inhérentes au processus d'innovation et engendre un processus créatif. Le processus transitionnel offre « un espace de questionnement, un travail de confrontation, de priorisation, mais aussi de dégagement par l'invention de nouveaux buts, de nouvelles valeurs. Ces inventions vont permettre de faire tenir ensemble des choses qui a priori ne tiennent pas ensemble, mais permettent donc de maintenir notre unité [...] et créent des liens inédits entre des représentations, des affects et des expériences " (Almudever, 2015). Dans cette expérimentation, ces nouveaux liens concernent l'ensemble des acteurs, quelle que soit leur place dans l'organisation, et favorisent la connexion entre les dimensions locale et stratégique tout comme entre la prescription et le réel de l'activité. Concernant les effets sur la santé, même si un net recul de l'absentéisme a été observé, il semble important de rester prudent dans la mesure où nombre de travaux ont montré que le simple fait d'intervenir dans un milieu et de solliciter ses acteurs peut aboutir de tels résultats (voir l'expérience Hawthorne notamment). D'autres évaluations nous semblent donc nécessaires sur ce point.

\subsection{Un cadre dialogique permettant de résoudre les problèmes}

Les résultats montrent de nouvelles voies pour soutenir le passage allant du diagnostic à la transformation, allier performance et santé, tout en entendant les besoins/appels des entreprises à aller vers plus de créativité et d'innovation. Cela exige de proposer des dispositifs susceptibles d'accompagner ces mouvements tout en conservant une place centrale à l'activité (Ughetto, 2018) et la confrontation entre les différents points de vue sur le travail. En ce sens, l'un des intérêts du DIT est de proposer un cadre dialogique qui permet aux acteurs de réguler eux-mêmes les tensions inhérentes à l'activité, notamment en offrant un cadre sécurisant pour confronter les process et décisions stratégiques, au travail réel et au contexte. En effet, de nombreux auteurs soulignent le caractère opératoire de la discussion sur la transformation du métier (Clot \& Faïta, 2000) et de l'organisation dans son ensemble (Detchessahar, Gentil, Grevin, \& Stimec, 2015). Pour autant, si cette discussion est un préalable à la santé et à la transformation, elle ne constitue pas une solution à elle seule. Elle met en évidence la complexité du travail, l'ensemble des tensions qui traversent l'activité, mais aussi des contraintes que l'environnement fait peser sur elle. Aussi, la discussion expose les acteurs à de nouvelles tensions, au moins durant un temps. Cela interroge donc sur les ressources à posséder pour supporter ces coûts, mais aussi sur ce qu'il y aurait à faire lorsqu'elles sont absentes. La littérature est en effet relativement discrète sur ces questions. Le DIT se positionne différemment puisqu'il se préoccupe d'emblée de ces coûts et propose un cadre pour les traverser. Ainsi, il constitue une opportunité de développement des ressources psychosociales. Rappelons en effet que la vitalité du métier provient d'interactions dynamiques entre ses diverses composantes (Bobillier Chaumon \& Sarnin, 2012) et nécessite donc des ressources psychosociales et un environnement capacitant (Falzon, 2005). Par ailleurs, sa méthodologie déplace l'objet de travail. Il ne s'agit plus directement pourrait-on dire, d'étudier l'activité pour la transformer, mais plutôt de «mettre en forme » une volonté de la transformer et de 
travailler cette forme au travers de l'étude de l'activité et en l'y confrontant. La discussion sur l'activité est un moyen au service du processus d'innovation organisationnelle et de l'activité transitionnelle, et permet à des acteurs aux métiers différents de partager une expérience commune de transformation des liaisons existant entre chacun d'entre eux et à différents niveaux de l'organisation. Ce processus semble relever d'une (re) création de liens dynamiques entre plusieurs métiers constituant le social de l'entreprise. Ce qui se joue, au sens winnicottien, n'est alors pas seulement les tensions entourant un métier, mais bien celles existant entre les métiers. Il en résulte, comme l'expérimentation du DIT semble le montrer, un renforcement du caractère opérant du processus d'intervention. En ce sens, on pourrait définir comme indicateur d'un processus d'innovation transitionnelle, des sujets de discussions qui ne portent plus uniquement sur un métier, mais sur les liens entre plusieurs métiers et plusieurs niveaux de l'organisation, qui aboutissent non pas à la transformation d'un métier, mais des liaisons existant entre plusieurs métiers situés à plusieurs niveaux de l'organisation. La pertinence de cette orientation se trouve renforcée par sa cohérence avec des travaux menés en ergonomie. En effet, dans un article récent, Bationo-Tillon, Poret et Folcher (2020) affirment qu'au cours du développement d'une organisation, «mondes du sujet, collectifs, sujets, systèmes d'activités se transforment de concert. Les limites bien établies de ces frontières disparaissent, car il ne faut pas s'y tromper, ce sont bien les relations entre ces entités qui se transforment » (p. 2). Ils proposent ainsi de réfléchir à l'élaboration de cadres théoriques permettant « de rendre compte de ce qui émerge dans l'entrelacs des systèmes d'activités » (ibid, p. 2). Mobilisant les travaux de Akkerman \& Bakker (2011), ils proposent de penser le développement comme un franchissement de frontières entre deux systèmes d'activité hétérogènes et s'intéressent aux «stratégies et activités de réparation/reconstitution/réassemblage mises en œuvre par les personnes qui œuvrent aux frontières» (ibid, p. 3). Et c'est justement aux processus transitionnels théorisés par Winnicott qu'ils font appel pour appréhender ce travail de (re)liaison. Ils créent le concept «d'instrument transitionnel » qui « ne contient pas uniquement l'idée de médiation [...], mais assure la fonction de lien, de transition entre une situation spatiotemporelle et une autre situation spatiotemporelle» (ibid, p. 6). Ainsi, la poursuite des recherches sur le DIT gagnerait à s'enrichir de ces travaux notamment en utilisant l'instrument transitionnel pour mieux saisir ce qui se joue entre les différents espaces et étapes du processus d'intervention. Parallèlement, il semble que le DIT constitue bien une tentative pour aller «au-delà des instruments transitionnels [vers] une approche transitionnelle [pouvant] appréhender (examiner, accompagner, co-concevoir) des dispositifs transitionnels au sein des organisations" (ibid., p. 23). Pour ce faire, et particulièrement lorsque les liaisons entre les métiers et les niveaux de l'organisation sont brisées, le DIT accompagne l'ensemble des mouvements affectifs auxquels les sujets et les collectifs sont exposés. L'activité transitionnelle ne peut en effet s'appréhender qu'en en considérant la seule dimension instrumentale (relier des systèmes d'activité hétérogènes). L'activité transitionnelle consiste bien à faire usage d'un objet transitionnel qui, en résistant aux attaques et en supportant toute une palette d'affects allant de l'agressivité à la contribution et à la sollicitude (Winnicott, 1999/1948), transforme le sujet. Ainsi, il serait nécessaire d'ajouter une dimension affective à l'instrument transitionnel pour tenter de saisir ce qui se joue et/ou se rejoue. 


\section{Conclusion}

Le DIT amène à considérer autrement l'enjeu principal d'une intervention : il ne s'agit plus d'analyser des données ou de révéler des phénomènes, quelles que soient la pertinence et la précision de l'analyse. L'enjeu prioritaire est ici, de proposer un processus en mesure de soutenir des acteurs - qui sont avant tout des sujets - engagés dans une activité de transformation de leurs activités et de leur organisation. Le DIT est conçu pour générer, accompagner et organiser cette activité de transformation, de sorte qu'elle aboutisse, s'implante, permette de considérer l'activité, protège et développe la santé. En ce sens, au sein du DIT, l'intervenant quitte franchement la posture d'expert: le dispositif ne comprend pas d'étape de diagnostic préalable aux préconisations et n'amène jamais l'intervenant à formuler de préconisations. En dehors de la phase d'étude de l'activité, son rôle est en effet de proposer un cadre permettant aux acteurs internes de transformer leurs propres situations de travail et organisation, puis d'animer les échanges de sorte que s'instaure une aire transitionnelle. Finalement, le DIT est un modèle d'intervention permettant aux entreprises d'aller vers une autoorganisation, sans faire fi des vécus associés à de telles innovations et en empêchant la non-considération de l'activité.

\section{BIBLIOGRAPHIE}

Akkerman, S., \& Bakker, A. (2011). Boundary Crossing and Boundary Objetcs. Review of Educational Research, 81(2), 132-169.

Alter, N. (1996). Sociologie de l'entreprise et de l'innovation. Paris : PUF.

Alter, N. (2000). L'innovation ordinaire. Paris : PUF.

Amado, G. (2007). Postface. In J.L. Prades (Ed.), Intervention participative et travail social (pp. 167-171). L'Harmattan.

Amado, G. (2015). Aux sources intimes de la mètis : espace potentiel, psychosocialité, résilience. Éducation Permanente, 202, 17-32.

Amado, G., \& Lhuilier, D. (2012). L'activité au cœur de l'intervention psychosociologique. Bulletin de psychologie, 519, 263-276.

Almudever, B. (2015). Créativité, création et personnalisation au travail. Présenté au colloque Travail et Créativité. Paris, CNAM, 19-20 novembre.

Bationo-Tillon, A., Poret, C., \& Folcher, V. (2020). Appréhender le développement des organisations à la croisée du cours d'action et de l'approche instrumentale : la perspective transitionnelle. Activités, 17(2). https://journals.openedition.org/activites/5371

Battistelli, A. (2012). L'innovation continue dans les organisations comme stratégie pour faire face aux transformations du travail et des organisations. Présenté au $17^{e}$ Congrès de l'Association Internationale de Psychologie du Travail de Langue Française. Lyon. 10-13 juillet. 
Battistelli, A. (2014). La recherche psychosociale sur le processus d'innovation individuelle et d'équipe : un bilan des connaissances. Psychologie du Travail et des Organisations, 4, 336-352.

Battistelli, A., \& Picci, P. (2008). Caractéristiques et processus d'une équipe de travail innovante : l'analyse d'un cas. In C. Lemoine, A. Balikdjian, N. Kridis, \& P. Salengros (Eds.), Psychologie du travail et développement des personnes et des organisations. Actes du $14^{e}$ Congrès de Psychologie du Travail et des Organisations (pp. 188-196). Éditions de l'AIPTLF.

Becque, M., Mauroux, A., Baradji, E., \& Dennevault, C. (2017). Quelles sont les évolutions récentes des conditions de travail et des risques psychosociaux ? Dares Résultats, $\mathrm{n}^{\circ} 082$. https:// dares.travail-emploi.gouv.fr/dares-etudes-et-statistiques/etudes-et-syntheses/dares-analysesdares-indicateurs-dares-resultats/article/quelles-sont-les-evolutions-recentes-des-conditionsde-travail-et-des-risques

Biron, C., Karanika-Murray, M., \& Cooper, C. L. (2012). Improving organizational stress and wellbeing interventions: Implications for theory, method, and practice. International Journal of Stress Management, 21(1), 85-111.

Biron, C., St-Hilaire, F., Baril-Gingras, G., Paradis, M.E., Chabot, S., Lefebvre, R, et al. (2016). Conditions facilitant l'appropriation de démarches préventives en santé psychologique au travail par les gestionnaires. Rapport de recherche R-921, IRSST. https://www.irsst.qc.ca/publications-et-outils/ publication/i/100867/n/demarches-preventives-sante-psychologique-au-travail

Bobillier Chaumon, M.E., \& Sarnin P. (2012). Manuel de Psychologie du travail et des organisations. Les enjeux Psychologiques du travail. De Boeck.

Bonnefond, J.Y., \& Clot, Y. (2016). Les affects et leur destin dans l'intervention. Activités, 13(2), $1-24$.

Brun, J. P., Biron, C., \& St-Hilaire, F. (2007). Guide pour une démarche stratégique de prévention des problèmes de santé psychologique au travail. http://www.irsst.qc.ca/media/documents/pubirsst/ rg-618.pdf

Caillé, A., Courtois, N., Galharret, J.M., \& Jeoffrion, C. (2020). Influence du leadership habilitant sur le bien-être au travail et l'engagement organisationnel : Étude comparative entre une organisation « habilitante » et une organisation « classique ». Psychologie du Travail et des organisations, 26(3), 247-261.

Caillé, A., \& Jeoffrion, C. (2017). Prévention des risques psychosociaux au sein d'établissements publics d'enseignement agricole : quand le diagnostic organisationnel participe d'une amélioration de la qualité de vie au travail. Psychologie du Travail et des Organisations, 23(4), 308-325.

Clot, Y. (1995). Le travail sans l'homme? Pour une psychologie des milieux de travail et de vie. La découverte.

Clot, Y. (2006). Clinique du travail et clinique de l'activité. Nouvelle revue de psychosociologie, 1, 165-177.

Clot, Y. (2014). Réhabiliter la dispute professionnelle. Le journal de l'école de Paris du management, 1(105), 9-16.

Clot, Y. (2017). Travail et pouvoir d'agir. PUF.

Clot, Y. (2018). Dialoguer pour faire autorité. In M. Detchessahar (Ed.), L'entreprise délibérée : refonder le management par le dialogue (pp. 7-19). Nouvelle Cité.

Clot, Y. (2019). La qualité de vie au travail est une notion subjective. Travail et Sécurité, 808. 
Clot, Y. (2021a). Le prix du travail bien fait. La Découverte.

Clot, Y. (2021b). Pour une coopération conflictuelle sur le travail bien fait. Alternatives Économiques. https://www.alternatives-economiques.fr/yves-clot-une-cooperation-conflictuelletravail-bien/00098894

Clot, Y., \& Faïta, D. (2000). Genres et styles en analyse du travail. Travailler, 4, 7-42.

Clot, Y., \& Leplat, J. (2005). La méthode clinique en ergonomie et en psychologie du travail. Le Travail Humain, 4, 289-313.

Coutarel, F., Caroly, S., Vézina, N., \& Daniellou, F. (2015). Marge de manœuvre situationnelle et pouvoir d'agir : des concepts à l'intervention ergonomique. Le Travail Humain, 78(1), 9-29.

Cox, T., Taris, T., \& Nielsen, K. (2010). Organizational interventions: issues and challenges. Work \& stress, 24(3), 217-218.

Davezies, P. (2014). L'individualisation du rapport au travail : un défi pour le syndicalisme. ETUI Policy Brief, 3, 3-6.

Debard, A. (2015). La prise en compte des risques psychosociaux par les managers. Les processus soutenant l'action des encadrants en santé au travail. Thèse de doctorat, Université Lumière Lyon 2, http://theses.fr/2017LYSE2089

Dejours, C. (1998). Souffrance en France. Le Seuil

Dejours, C., \& Bègues, F. (2009). Suicide et travail : que faire? Paris : PUF.

Detchessahar, M. (2019). L'entreprise délibérée : refonder le management par le dialogue. Nouvelle Cité.

Detchessahar, M., Gentil, S., Grevin, A., \& Stimec, A. (2015). Quels modes d'intervention pour soutenir la discussion sur le travail dans les organisations ? Réflexions méthodologiques à partir de l'intervention dans une clinique. @GRH, 16, 63-89.

Devereux, G. (1967). De l'Angoisse de la Méthode dans les Sciences du Comportement. Flammarion.

Dugué, B., Petit, J., \& Daniellou, F. (2010). L'intervention ergonomique comme acte pédagogique. Perspectives Interdisciplinaires sur le Travail et la Santé, 12-3, 1-26.

Falzon P. (2005). Ergonomics, knowledge development and the design of enabling environments. In Humanizing Work and Work Environment Conference (pp. 10-12).

Fuchs, C., Jeoffrion, C., Barré, S., \& Savary, F. (2018). La qualité de vie au travail dans les établissements sanitaires et médico-sociaux : Co-construction d'une méthodologie d'enquête pilotée par la MiRH via un processus participatif. Perspectives interdisciplinaires sur le travail et la santé, 20(2).

Giusto, B. (2014). L'innovation en entreprise. Les dégâts du prêt à penser des recettes technicogestionnaires et des approches managériales. In J.P. Durand, F. Moatty, \& G. Tiffon (Eds.), L'innovation dans le travail (pp. 23-34). Editions Octarès.

Goulet F., \& Vinck, D. (2012). Innovation through Withdrawal. Contribution to a Sociology of Detachment. Revue Française de Sociologie, 53(2), 117-146.

Guidou, N. (2017). La qualité, l'innovation et la créativité du travail au sein des organisations : vers une politique globale de prévention de la souffrance psychique au travail. Thèse de doctorat, Université Lumière Lyon 2, http://theses.fr/2017LYSE2089 
Guidou, N. (2019). Agir en faveur de la qualité de vie au travail : présentation d'une rechercheaction innovante étudiant les interactions entre acteurs internes et processus d'intervention. Psychologie du Travail et des Organisations, 25, 75-85

Grosjean, V., \& Guyot, S. (2016). Bien-être et qualité de vie au travail, risques psychosociaux : de quoi parle-t-on? Hygiène et Sécurité Du Travail, 242.

Habermas, J. (1987). Théorie de l'agir communicationnel. Tome 1. Fayard.

Hansez, I., Bertrand, F., \& Barbier, M. (2009). Évaluation des pratiques de diagnostic de stress au sein d'entreprises belges : facteurs bloquants et facteurs stimulants. Le Travail Humain, 72(2), 127-153.

Hansez, I., \& Keyser de, V. (2007). Du diagnostic des risques psychosociaux à la gestion organisationnelle du stress. In M. Neboit, \& M. Vézina (Eds.). Stress au travail et santé psychique (pp. 189-206). Octarès.

Kanter, R. (1988). When a thousand flowers bloom: structural, collective, and social conditions for innovation in organizations. In B.M. Staw, \& L.L. Cummings (Eds.), Research in organizational behavior (p. 169-211). Greenwich, CT : JAI Press.

Levet. P. (2013). Des risques psychosociaux à la qualité de vie au travail. Équiper les acteurs pour négocier le travail, l'expérience de l'ANACT. Négociations, 19, 97-111.

Lhuilier, D., \& Roche, P. (2009). Introduction. Nouvelle revue de psychosociologie, 7(1), 7-18.

Mallein, P., \& Toussaint, Y. (1994). L'intégration sociale des technologies d'information et de communication. Une sociologie des usages. Technologies de l'information et société, 4, 315-335.

Moatty, F., \& Tiffon, G. (2014). Sociologie du travail et sociologie de l'innovation. Un rendez-vous manqué ? In J.P. Durand, F. Moatty, \& G. Tiffon (Eds.). L'innovation dans le travail (pp. 1-11). Octarès.

Peters, S., Faulx, D., \& Hansez, I. (2010). Le rôle des objets-frontière dans le découpage temporel et social d'une innovation de service. Étude de cas d'un transfert de technologie depuis un laboratoire universitaire de sciences sociales. Revue d'Anthropologie des connaissances, 4, 65-86.

Prades, J. L. (2012). Groupes d'usagers, souffrance et créativité. Autour de trois interventions sociopsychanalytiques. Bulletin de psychologie, 519, 239-249.

Reille-Baudrin, E. (2011). Reconversion professionnelle, l'espace d'une transition : d'une clinique de l'expérience à l'expérimentation clinique de l'activité transitionnelle : la méthode des instructions au sosie. Thèse de doctorat, CNAM, Paris. http://theses.fr/2011CNAM0781

Rouat, S., \& Sarnin, P. (2013). Prévention des risques psychosociaux au travail et dynamique de maturation : le processus d'intervention comme opérateur de la transformation et du développement de la coopération. Activités, 10(1), 58-72. https://journals.openedition.org/ activites $/ 548$

Rouat, S., \& Sarnin, P. (2018). L'intervention en santé au travail : l'intérêt de l'étude de cas d'intervention. Le Travail Humain, 81, 331-361.

Sarnin, P., Caroly, S., \& Douillet, P. (2011). Contre les « risques » psychosociaux, quel débat sur l'activité ? Le Travail Humain, 74(4), 309-320.

Sarnin, P., Durif-Bruckert, C., \& Rouat, S. (2011). Le rapport à la santé des travailleurs : contexte et stratégie. Psihologia Resurcelor Umane, 9(2), 72-89. 
Star, S.L., \& Griesemer, J.R. (1989). Institutional ecology, translations and boundary objects: amateurs and professionals in Berkeley's Museum of Vertebrate Zoology, 1907-39. Social Studies of Sciences, 19, 387-420.

Supiot, A. (2017). Et si l'on refondait le droit du travail. Le Monde diplomatique, (763), 1-22.

Sznelwar, L., \& Le Doaré, H. (2006). Alain Wisner : Le développement de l'ergonomie et de la pensée sur le « travailler ». Travailler, 1(1), 39-54.

Ughetto, L. (2018). Organiser l'autonomie au travail : Travail collaboratif, entreprise libérée, mode agile. FYP.

Valléry, G., \& Leduc, S. (2012). Les Risques Psychosociaux. PUF.

West, M.A., \& Farr, J.L. (1990). Innovation at work. In M.A. West, \& J.L. Farr (Eds.). Innovation and creativity at work: Psychological and organizational strategies (pp. 3-13). Wiley.

Winnicott, D. W. (1971). Jeu et réalité. Gallimard.

Winnicott, D. W. (1999/1948). Le premier contact avec la réalité externe. L'enfant, la psyché et le corps. Payot.

Winnicott, D. W. (2000/1969). L'usage de l'objet et le mode de relation à l'objet au travers des identifications. La crainte de l'effondrement et autres situations cliniques. Gallimard.

Winnicott, D. W. (2002/1953). Objets transitionnels et phénomènes transitionnels. Jeu et réalité. L'espace potentiel. Gallimard.

\section{NOTES}

1. La relation à l'objet relève de l'expérience subjective du sujet tandis que l'utilisation de l'objet est ancrée dans l'expérience vécue.

2. Si la validation a été officielle en juillet, le lancement était prévu pour septembre, l'institut de formation fermant l'été.

3. Pas de management intermédiaire dans cet établissement.

4. L'appel à volontariat concerne chaque groupe métier. Dans ce contexte, auprès des formateurs et auprès des fonctions transversales.

5. Cette fonction a été explicitée lors des étapes de présentation et de validation du DIT. Elle a fait l'objet d'une communication orale et écrite au travers de documents créés pour la démarche.

6. Temps 1 : formation aux bases de l'analyse de l'activité puis aux méthodes identifiées par l'intervenant pour analyser l'activité

- Temps 2 : étude des activités réalisée avec l'intervenant

- Temps 3: analyse des données recueillies avec l'intervenant et préparation du travail de restitution au COPIL.

7. Voir Guidou (2017) pour obtenir le détail des projets. 


\section{RÉSUMÉS}

Si les démarches dites de qualité de vie au travail (QVT), selon leur dénomination politique, sont décrites comme recherchant l'alliance entre les performances économiques et les performances sociales, elles suscitent encore nombre de réserves. En effet, pour que la QVT ne soit pas qu'un "glissement lexical» de plus dans le champ de la santé psychique au travail (Clot, 2019), il est nécessaire de se poser sérieusement la question des méthodologies à construire afin qu'elle réponde « aux problématiques socio-organisationnelles qui naissent de la vie de l'entreprise [...] et non qu'elle vienne en surplomb constituer un énième projet à ajouter [...]» (Levet, 2013, p. 104). Dans cette perspective, l'objectif de cet article est de présenter une méthodologie reposant sur des innovations organisationnelles générées à partir d'activités transitionnelles sources de créativité et de santé (Lhuilier \& Roche, 2009; West \& Far, 1990; Winnicott, 1999/1948). Ce dispositif, dit d'innovation transitionnelle, vise ainsi particulièrement à dépasser la difficulté à aller du diagnostic à la transformation durable et à accompagner les acteurs dans les épreuves à traverser. Il a été expérimenté pour la première fois dans un service de santé au travail qui devait intervenir au sein d'un institut de formation supérieur aux prises avec d'importants troubles psychosociaux. L'analyse de ses résultats a permis d'observer des transformations de l'organisation et des situations de travail et leur durabilité un an après la clôture du dispositif.

While according to their political name so-called quality of working life (QWL) approaches are described as seeking an alliance between economic performance and social performance, they still give rise to a number of reservations. Indeed, if QWL is not to be just another "lexical shift" in the field of mental health at work (Clot, 2019), it is necessary to seriously consider the question of the methodologies to be developed in order for it to respond "to the socio-organisational problems that arise in the life of the company [...] and not to be an overhang constituting yet another project to be added [...]" (our translation, Levet, 2013, p. 104). In this perspective, the objective of this article is to present a methodology based on organisational innovations generated from transitional activities that are sources of creativity and health (West \& Far, 1990; Winnicott, 1999/1948; Lhuilier \& Roche, 2009). This approach, known as transitional innovation, specifically aims at overcoming the difficulty of moving from diagnosis to sustainable transformation and at accompanying the actors through the trials and tribulations of the process. It was tested for the first time in an occupational health department that was to intervene in a higher education institute that was experiencing major psychosocial problems. The analysis of its results made it possible to observe transformations in the organisation and work situations and their sustainability one year after the end of the scheme.

\section{INDEX}

Mots-clés : méthodologie, qualité de vie au travail, transformation organisationnelle, innovation, activité transitionnelle

Keywords : methodology, quality of working life, organizational transformation, innovation, transitional activity 


\section{AUTEURS}

\section{NADĖGE GUIDOU}

Université Lumière Lyon 2, Laboratoire GREPS, 5 avenue Pierre Mendes France, 69500 Bron n.guidou@gmail.com

\section{PHILIPPE SARNIN}

Université Lumière Lyon 2, Laboratoire GREPS, 5 avenue Pierre Mendes France, 69500 Bron, philippe.sarnin@univ-lyon2.fr 